ENCYCLOPEEDIE Encyclopédie berbère

BERBERE

32 | 2010

32 | Mgild - Mzab

\title{
Mil (Monde touareg)
}

M. Gast

\section{OpenEdition}

Journals

Édition électronique

URL : https://journals.openedition.org/encyclopedieberbere/606

DOI : 10.4000/encyclopedieberbere.606

ISSN : 2262-7197

\section{Éditeur}

Peeters Publishers

\section{Édition imprimée}

Date de publication : 31 décembre 2010

Pagination : 5024-5028

ISBN : 978-90-429-2369-0

ISSN : 1015-7344

\section{Référence électronique}

M. Gast, « Mil (Monde touareg) », Encyclopédie berbère [En ligne], 32 | 2010, document M116c, mis en ligne le 11 novembre 2020, consulté le 17 février 2022. URL : http://journals.openedition.org/ encyclopedieberbere/606; DOI : https://doi.org/10.4000/encyclopedieberbere.606

Ce document a été généré automatiquement le 17 février 2022.

(C) Tous droits réservés 


\section{Mil (Monde touareg)}

\section{Gast}

1 Le mil est une céréale des zones tropicales africaines et sahariennes de la famille des Graminées (Poacées). Cependant les mils ne correspondent pas à une entité botanique précise et ne peuvent être scientifiquement définis.

2 L'imprécision de ce terme a pour conséquence l'attribution du nom de mil à diverses plantes qui parfois ne sont même pas des Graminées.

3 Le sarrazin appelé «millet noir» est une Polygonacée. Le fonio, petite Graminée de cueillette, est classée sous l'appellation de Paspafolium longiflorum ou encore de Digitaria exilis, « petit mil » ou « mil d'Egypte» (cf. Adrian, 1954, p. 21).

4 Dans le sud-ouest de la France, les cultivateurs appellent parfois mil, le maïs ordinaire, et en Poitou-Charentes le millet, de culture ancienne, est souvent appelé mil.

5 Les botanistes ont l'habitude de considérer comme mils les espèces suivantes et les variétés qui en découlent : Panicum, Pennisetum, Setaria, Eleusine, Paspalum (voir Portères, Bulletin de l'IFAN, 1951, 13, 1-78; et Adrian 1954, p. 23-24).

6 Nous retiendrons l'espèce Pennisetum qui est celle cultivée et consommée au Sahara central (pays touareg), mais dont le plus gros fournisseur au Niger est la région du Damergou. Appelé souvent petit mil, ou pénicillaire; il comporte plusieurs variétés affectées de noms locaux spécifiques selon les pays (Angleterre, Allemagne, Indes, Arabie). En Afrique ses appellations varient d'une région à l'autre (par exemple au Sénégal, il existe une dizaine de noms différents).

7 En pays touareg, le mil chandelle (Pennisetum) est appelé énélé (absis en kabyle) et duknhn en arabe du Proche-Orient; alors que cette appellation est ignorée en Afrique du Nord où le mot bešna est attribué aux graines de sorgho confondu éventuellement avec celles de mil (cependant très rares en Afrique du Nord).

8 Le mil chandelle de l'espèce Pennisetum est appelé aussi Typhoïdum ou Spicatum, Americanum ou Glaucum.

9 Les consommateurs de mil (surtout en bouillie) différencient les mils du Fezzan, de Djanet, de l'Ahaggar*, d'Agadez, de Tegedda-n-Tésemt, de Tahoua ou du Damergou, selon leur goût mais aussi selon la longueur des épis, la densité de leurs graines, la 
présence ou l'absence de poils entre les graines. Les semailles du mil peuvent commencer fin mars, et il parvient à maturité en trois mois. Mais ces semailles peuvent se pratiquer jusqu'à la fin novembre, c'est-à-dire quasiment toute l'année, sauf durant les trois mois les plus froid: décembre, janvier, février. A Idelès* (Ahaggar), les cultivateurs peuvent semer trois fois du mil, d'avril jusqu'en septembre, soit dans des carrés exclusivement réservés au mil, soit le long des rigoles d'arrosage d'autres cultures. Ainsi les pieds de mil suffisamment arrosés peuvent être moissonnés de quatre à cinq fois. Après les premiers épis réservés à la consommation humaine, les autres récoltes sont accordées aux animaux en fanes vertes, épis séchés et tiges.

En 1965, nous avons établi en collaboration avec Jean Adrian la valeur nutritionnelle des différents procédés de mouture des mils et du sorgho.

11 Pour comprendre les caractéristiques des moutures qu'on améliore par le blutage, il faut rappeler la structure générale d'un grain de céréale qui comporte quatre fractions distinctes anatomiquement et biochimiquement :

- Le péricarpe, enveloppes ou sons, qui renferme la majeure partie de la cellulose et autres glucides hautement polymérisés (cellulose brute).

- Les assises protéiques ou couche merveilleuse ou encore couche d'aleurone contenant un certain nombre de couches anatomiquement distinctes qui varient selon les céréales. Elles contiennent en quantité abondante des protéines et des vitamines B ainsi que certains oligominéraux. Néanmoins leur paroi cellulaire étant fort résistante, la mouture doit être très poussée pour que le contenu de ces cellules soit utilisable par l'homme.

- Le germe ou embryon possède, avec les assises protéiques, le privilège d'être la fraction la plus intéressante nutritionnellement. Il est riche en protéines et en vitamines B. Les lipides sont concentrés dans cette fraction du grain.

- Enfin l'endosperme ou amande du grain représente la fraction la plus importante au point de vue pondéral. Elle renferme un pourcentage important d'amidon, mais reste assez pauvre en vitamines et sels minéraux ; les protéines quelle contient sont de qualité médiocre. (D'après Adrian in Gast et Adrian 1965, p. 41). «La justification physiologique du blutage est l'élimination des constituants cellulosiques du grain, ce qui entraîne une amélioration de la valeur organoleptique du produit céréalier ainsi que son utilisation digestive.... » (Idem, p. 42).

Durant les années soixante, les techniques de mouture des mils n'ont guère intéressé les Occidentaux car la politique d'expansion de la production et de la consommation du blé représentait un axe très puissant sur les plans géostratégique et financier. Les premiers essais mécaniques du broyage des grains de mil furent très décevants. Car pour séparer le péricarpe indigeste de l'amande du grain, il fallait le mouiller et ensuite le vanner. Cette opération était traditionnellement réalisée par les Africains dans le grand mortier de bois avec un gros pilon frappant vigoureusement les grains durant environ dix minutes. Pour un litre de mil légèrement humide, il faut environ 200 coups de pilon, monder rapidement l'ensemble à l'aide d'un van de paille tressée, et remettre une deuxième fois dans le mortier les graines humides pour les concasser encore (avec 100 nouveaux coups de pilon) afin d'obtenir une mouture qui comporte alors trois granulométries : de la farine, des concassures moyennes et des grosses concassures qui présentent encore quelques graines presque entières.

Ces trois granulations sont importantes pour la cuisson de la bouillie appelée ésink en tamâhaq (ou éšink en tawellemmet; عșida en arabe). La ménagère qui a préparé une marmite de porterie locale remplie d'eau bouillante, verse d'abord les grosses 
concassures qui cuisent ainsi vingt minutes à feu vif et sont sans cesse remuées à l'aide d'une palette de bois. La marmite dégorge au début une mousse jaunâtre écumée aussitôt et jetée à terre. Quand la mousse devient blanche, la bouillie est encore remuée environ vingt minutes, et la ménagère y rajoute les concassures moyennes avec un peu d'eau chaude. Après dix minutes de cuisson, elle verse en pluie fine la farine en remuant vigoureusement le tout formant un magma collant qui adhère vite au récipient. De grosses bulles éclatent à la surface de la bouillie; la marmite retirée du feu au bout de cinq minutes est mise en attente une demi-heure avant d'être consommée.

14 Cette bouillie est douce au goût comme au palais. Son aspect granuleux de couleur gris vert indique la bonne qualité de la préparation. Elle est alors versée dans un grand plat de bois noirci au feu appelé tayehut et agrémentée soit d'un peu de beurre fondu, soit de lait aigri disposé dans un creux au milieu du plat. Chaque convive prend avec sa cuillère en bois (tesûkalt) un peu de bouillie avec du lait en respectant la cadence collective et en puisant toujours au même endroit en face de soi. Le mil humidifié, mondé et pilé à nouveau doit être vite préparé car il s'oxyde vite et devient amer.

Alors que la bouillie est le plat traditionnel en pays touareg, le mil peut se consommer de multiples façons ; cru, en graines rôties, en crêpes, en galettes, en boisson, en soupe ou même en couscous (surtout dans les pays du Sahel). Un homme affamé peut croquer des graines de mil cru (teyzaz ou agazaz) ou absorber une mouture entre deux pierres (tamazatt), sinon en poudre avec d'autres céréales, des dattes, ou des cœurs de plantes sauvages (tébik). Si l'on mouille les grains en les faisant tremper dans l'eau (au moins une heure) c'est tédéwt, tegra, ngaji ou titefferatïn quand les épis encore verts sont rôtis sous la cendre chaude et frottés entre les mains (voir Gast 1968, p. 104, et 1965, p. 32).

Les moutures de mil entrent souvent dans la confection de breuvages froids utilisés en voyage. C'est surtout l'aygera mélange d'eau, de fromage pilé, de mouture de mil à laquelle l'on peut rajouter des dattes pilées et un peu de piment. Cette boisson rafraîchissante, très roborative, est appréciée l'été par les méharistes qui la transportent dans une outre verticale suspendue au pommeau de leur selle (Gast 1968, p. 73 et Bernus 1981, p. 211).

17 Ces recettes peuvent aussi se pratiquer avec des graines sauvages telles que le Panicum Laetum ou le cenchrus biflorus (cram-cram).

18 Comment arriver à épargner le travail pénible et fastidieux aux femmes africaines, tout en sauvegardant les qualités gustatives et nutritionnelles du mil ? Pour essayer de répondre à cette question, nous nous sommes livrés à de minutieuses analyses biochimiques comparatives selon les modes de broyages et selon l'origine des grains. Les instruments de broyage des grains couramment utilisés en Ahaggar étaient: la meule dormante (surface plane immobile sur laquelle l'on frotte un broyeur de pierre, tašafart en tamâhaq), la meule tournante (moulin de pierre rotatif), le mortier de bois.

19 «En comparaison avec les moutures très rudimentaires sur meules de pierre, le traitement au mortier de bois offre l'avantage d'éliminer la majeure partie des éléments cellulosiques néfastes à la digestibilité d'une ration....

20 Ainsi, le traitement au mortier de bois représente une sorte de technique optimum, éliminant les matières indésirables tout en conservant les éléments utiles au grain » (Adrian, 1965, p. 62, voir tableau II en annexe). 
21 La recherche sur le décorticage des mils s'est d'abord effectuée à l'aide de moulins déjà existants pour le café, le riz ou le maïs. Depuis les essais de moulin à traction animale, on est passé à ceux entraînés par un moteur comme celui du broyeur à marteaux (voir schéma et fonctionnement de ce broyeur in François 1988, p. 71 et suivantes). La mécanisation du broyage du grain doit commencer sur un grain sec et, après blutage, moudre ce grain débarrassé de son enveloppe indigeste. D'autres problèmes se sont alors posés ; l'apprentissage de cette technique, l'adaptation aux petites communautés villageoises qui demandent de faibles quantités, les prix de revient et les goûts et qualités de ces moutures, etc. Quarante années se sont écoulées avant de parvenir à concevoir de grosses unités de productions dans les pays africains (Sénégal, BurkinaFaso, Niger, Mali, etc.) et de donner au mil son rang comme céréale à part entière en Afrique et dans le commerce international des grains.

Le mil est une céréale très demandée par les consommateurs africains à tel point, qu'avec l'augmentation démographique, les pays producteurs (Niger, Mali) ont dû interdire l'exportation du mil par caravanes (sel contre mil, comme celles vers l'Algérie) ou par camion. Leurs productions ne suffisent pas toujours à nourrir leurs populations surtout après des années de sécheresse. Ainsi, la production du mil qui constituait une base d'échanges locaux très importante dans les pays du Sahel et au Sahara central est toujours insuffisante aujourd'hui ; le mil se voit donc concurrencer par les importations du riz, de blé, de maïs et même de sorgho.

Car la ration alimentaire pour un travailleurs africain ou saharien est de $560 \mathrm{~g}$ de grains environ (voir Gast, 1968, p. 304 et sq.). Cependant, chez les nomades cette ration peut être supplémentée par d'autres produits tels le fromage, le lait frais ou aigri, le beurre et des dattes, produits aléatoires certes qui peuvent varier énormément selon les saisons ou les lieux.

Après des analyses biochimiques très précises sur les laits, beurre et fromages, nous avons pu conclure : «... qu'une ration alimentaire composée de $1 \mathrm{~kg}$ de lait de chèvre entier, $1 \mathrm{~kg}$ de lait de chèvre baratté (aigri) et de $450 \mathrm{~g}$ de mil satisferait des besoins énergétiques du nomade saharien et, de plus, serait sensiblement équilibré sur le plan nutritionnel » (Gast, Maubois, Adda, 1968, p. 55).

\section{BIBLIOGRAPHIE}

Les études sur les mils et le sorgho sont très nombreuses ; ne sont mentionnées ici que celles citées dans le texte :

ADRIAN J., I, « Les mils et les sorghos. Valeur alimentaire, usage », in Les plantes alimentaires de l'ouest africain, Dakar, ORANA, 1954.

ADRIAN J. et Ch. SAYERSE, II, « Composition de mils et sorghos du Sénégal », Ibid., Dakar, ORANA, $1954,167 \mathrm{p}$.

ADRIAN J., JACQUOT R., Le sorgho et les mils en alimentation humaine et animale, Paris, Vigot éditions, 1964, $188 \mathrm{p}$. 
ALTERSIAL (Collectif), Du grain à la farine, le décorticage et la mouture des céréales en Afrique » de l'Ouest, Ministère de la Coopération et du Développement, Centre technique de coopération, Groupe de recherches et d'échanges technologiques agricole et rurale (CTA) (GRET), Paris, $1988 ; 279$ p.

ALTERSIAL, OCDE, CILSS, Mil, Maïs, Sorgho. Techniques et alimentation au Sahel (Collectif), Paris, L'Harmattan/Pusat, 1989, 171 p.

BERNUS E., Touaregs nigériens. Unité culturelle et diversité régionale d'un peuple pasteur, Paris, Mémoire de l'ORSTOM n ${ }^{\circ} 94,1981,508$ p. + 4 plans

CHANTEREAU J. et NICOU R., Le sorgho, A.C.C.I., C.I.A., Paris, Ed. Maisonneuve et Larose, 1991, 159 p.

GAST M., ADRIAN J. Mils et sorgho en Ahaggar, Etude ethnologique et nutritionnelle, Paris, AMG (Mémoire du CRAPE $n^{\circ}$ IV), 1965, 78 p.

GAST M., MAUBOIS J.-L., ADDA J., Le lait et les produits laitiers en Ahaggar, Paris, AMG (Mémoires du CRAPE $\left.\mathrm{n}^{\circ} \mathrm{XIV},\right), 1970,71 \mathrm{p}$.

PORTERES R., voir Bulletin de l'IFAN, 1951, 13, 1, Agronomie tropicale 1950, 489, 495.

\section{INDEX}

Mots-clés : Agriculture, Alimentation, Botanique, Economie, Mali, Préhistoire, Sahara, Sahel 